\title{
Quantum coherence and interaction-free measurements
}

\author{
Sierk Pötting ${ }^{1,2,3} *$, Eun Seong Lee ${ }^{1}$, William Schmitt ${ }^{1}$, Ilya Rumyantsev ${ }^{1}$, Bernd Mohring ${ }^{1}$ and Pierre Meystre ${ }^{1}$ \\ ${ }^{1}$ Optical Sciences Center, University of Arizona, Tucson, Arizona 85721 \\ ${ }^{2}$ Max-Planck-Institut für Quantenoptik, 85748 Garching, Germany \\ ${ }^{3}$ Sektion Physik, Universität München, 80333 München, Germany
}

(November 13, 2018)

\begin{abstract}
We investigate the extent to which "interaction-free" measurements perturb the state of quantum systems. We show that the absence of energy exchange during the measurement is not a sufficient criterion to preserve that state, as the quantum system is subject to measurement dependent decoherence. While it is possible in general to design interactionfree measurement schemes that do preserve that state, the requirement of quantum coherence preservation rapidly leads to a very low efficiency. Our results, which have a simple interpretation in terms of "which-way" arguments, open up the way to novel quantum non-demolition techniques.
\end{abstract}

PACS numbers: 03.65.Bz, 42.50.Ct, 03.67.-a

Interaction-free measurements (IFM) are a particularly puzzling example of the "paradoxes" illustrating the strangeness of quantum physics. In a nutshell, they are measurements that offer a way to detect the presence of an object without any apparent interaction with the measuring device.

The history of IFM can be traced back to 1960, when Renninger [2], realized that at the quantum level, the non-observation of a result does have a physical impact in that it implies a collapse of the wave function. This point was further investigated by Dicke [3] in the framework of non-scattering of photons by particles. In 1993, Elitzur and Vaidman (EV) proposed a measurement scheme illustrating particularly vividly the IFM paradox: They demonstrated that one can to a certain extent determine the presence of a classical or quantum mechanical object in an interferometer path without touching it with a probe photon, and without prior information about the location of the object. The EV scheme was soon experimentally realized [4] and its efficiency was subsequently increased by use of the quantum Zeno effect [5]. Recent research has been directed towards applications of the scheme, e.g. in the interaction-free imaging of macroscopic objects with less than the classically expected amount of light [6].

Of course, IFMs are not really interaction-free: if the interaction Hamiltonian between the object and the "measuring stick", in most experimental cases a light beam, were to be set equal to zero, then nothing would

*email: sierk.poetting@wotan.opt-sci.arizona.edu happen. But past this rather trivial and easy way out of the problem, a more interesting question is to try and quantify the meaning of interaction-free. "Energy exchange free" [7] is now well established as a more precise way to characterize IFMs in the case of classical objects. This concept has also been applied at the quantum level, where two-state systems have been investigated [1,8,9. In a recent paper, White et al [10] showed that true IFM is not possible in the optical detection of two-level atoms, due to the nonzero rate of forward scattered photons. This gives a first indication that the quantum mechanical situation is indeed more subtle than classical IFM.

It is well known that the quantum superpositions that oftentimes characterize the state of an object are more sensitive to interactions than its energy. In order to gain a full understanding of IFMs, it is therefore important to analyze their impact on such states. In addition, quantum superpositions constitute a major ingredient of quantum entanglement. As such they play a central role in quantum information processing, including teleportation [11], quantum computing [12,13] and cryptography 114,15. Decoherence is one of the major obstacles to quantum information processing, and the impact of IFMs on quantum coherence is an important question in this context.

The goal of this paper is to analyze the impact of IFMs on quantum coherence, as well as their interplay. Our main conclusion is that even "energy exchange free" is an overly simplified description of the situation. IFMs do generally change the state of the system, as evidenced e.g. in the destruction of the quantum superposition of two internal states as well as of the quantum entanglement of two atoms. However, we also show that carefully designed IFM schemes can provide a powerful and nondestructive tool to probe quantum superpositions and entanglements. Potential applications include the determination of the presence of ions in a linear trap without disturbing their entanglement, and hence an ongoing quantum computer calculation.

The situation that we consider is an extension of the EV scheme, considering a multilevel atom in a coherent superposition of electronic levels. Our goals are (a) to determine whether an IFM can detect the presence of an atom without destroying that superposition, and (b) to quantify the impact of that superposition on the outcome of the IFM measurements.

An essential ingredient of the EV scheme is the fact 
that if the photon path is along the arm of the interferometer where the object is located, it will be irreversibly absorbed by this object. Figure 1 illustrates a model atomic system that has this same property. It consists of a four-level atom, initially in a general superposition of the two metastable states $\left|m_{-}\right\rangle$and $\left|m_{+}\right\rangle$.

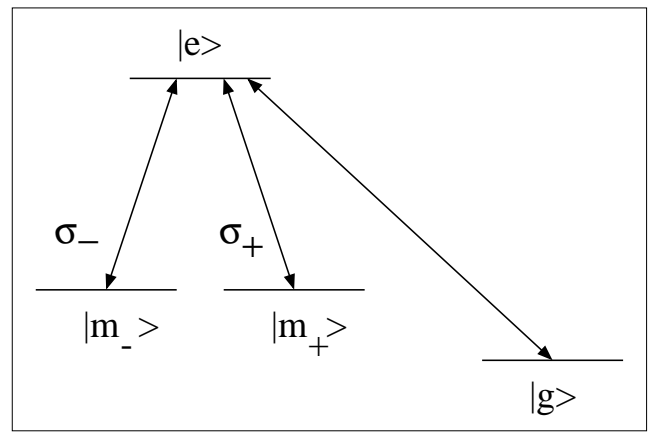

FIG. 1. The atomic level scheme: The metastable states $\left|m_{-}\right\rangle$and $\left|m_{+}\right\rangle$are coupled to the excited state $|e\rangle$ via circularly polarized light. The state $|e\rangle$ rapidly decays to a stable ground state $|g\rangle$ which is far off-resonance from the metastable states. Once in this state, the atom is transparent to the light in the interferometer path.

The atoms can absorb single photons with unit efficiency, inducing a transition to the excited level $|e\rangle$, from which they can irreversibly decay to the ground state $|g\rangle$. If the wavelength of the $|e\rangle \longleftrightarrow|g\rangle$ transition is much larger than that of the $\left|m_{ \pm}\right\rangle \longleftrightarrow|e\rangle$ transitions, then the branching ratio of these transitions is extremely high, and reabsorption can be neglected. As a result, the absorption of photons is de facto irreversible, the state $|g\rangle$ being the "exploding bomb" state of Ref. [1]. Filtering of the high-frequency spontaneous photons circumvents the problem of forward scattering described in Ref. [10].

As illustrated in Fig. 22, the IFM scheme of Ref. [1] is extended in such a way that the atom in the lower arm of the interferometer is in a superposition of the internal metastable states, the initial state of the atomic system taken for concreteness to be

$$
\left|\phi_{\text {atom }}\right\rangle=\frac{1}{\sqrt{2}}\left(\left|m_{+}\right\rangle+\left|m_{-}\right\rangle\right) \text {. }
$$

This superposition can be interpreted as describing an atom which is half absorbing and half transparent for a given photon polarization. While at first sight similar to the superposition states of being "there" or "not there" of Ref. [1.9], our "half-absorbers" are both located inside the interferometer path and thus the polarization dependence of the two transitions involved provides an additional degree of freedom. An appropriate choice of field polarization enables us to simultaneously probe both constituents of the superposition. This opens up the way to additional control on the outcome on the IFM, and in particular to the possibility of a non-demolition measurement of the quantum superposition of Eq. (11). As such, it permits the IFM of a "quantum bomb" in a superposition of its "armed" and "unarmed" states.

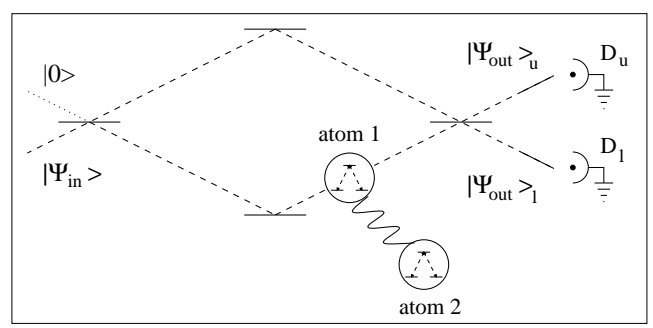

FIG. 2. "Interaction-free" measurement on multi-level atoms. In addition to single atoms in internal superpositions one can also consider two entangled atoms, only one of them being in the interferometer path.

We denote the photon creation and annihilation operators by $\hat{a}_{k, \mu}^{\dagger}$ and $\hat{a}_{k, \mu}$, respectively, where $k=\{u, l\}$ stands for photons of polarization $\mu$ following the upper or lower path in Fig. 2. Hence, the ket $\hat{a}_{l, \mu}^{\dagger}|0\rangle$ describes a photon of polarization $\mu$ in the lower interferometer arm. We consider both circular polarizations $\sigma_{ \pm}$, in which case $\mu=\{+,-\}$, and linear polarizations $\mu=\{x, y\}$, the atomic selection rules being such that the $\left|m_{-}\right\rangle \leftrightarrow|e\rangle$ and $\left|m_{+}\right\rangle \leftrightarrow|e\rangle$ are excited by left and right-circularly polarized light $\sigma_{-}$and $\sigma_{+}$, respectively. The absorption of a photon by the atom can therefore be described by the transition

$$
\hat{a}_{l, \pm}^{\dagger}|0\rangle\left|m_{\mu}\right\rangle \rightarrow\left\{\begin{aligned}
|\mathcal{S}\rangle|g\rangle & \text { for } \quad \mu= \pm, \\
\hat{a}_{l, \pm}^{\dagger}|0\rangle\left|m_{\mp}\right\rangle & \text { for } \quad \mu=\mp
\end{aligned}\right.
$$

where $|\mathcal{S}\rangle$ is a scattered photon. We assume that this high-frequency photon escapes the system without possible reabsorption, and can be additionally be filtered away from the detectors.

As in the original EV setup, a single photon enters the interferometer via the lower input port in the state

$$
\left|\psi_{\text {light }, i n}\right\rangle=a_{l, \mu}^{\dagger}|0\rangle \text {. }
$$

Two identical non-polarizing 50-50 beam splitters constitute the input and output of the interferometer. The phase shifts upon transmission are chosen so that the upper detector $D_{u}$ fires with unit probability in case if no atom is in the interferometer. In that case, the annihilation operators $\hat{a}_{u, l}$ before and $\hat{a}_{u, l}^{\prime}$ after each beam splitter are related by

$$
\left(\begin{array}{c}
\hat{a}_{u}^{\prime} \\
\hat{a}_{l}^{\prime}
\end{array}\right)=\frac{1}{\sqrt{2}}\left(\begin{array}{cc}
i & 1 \\
1 & i
\end{array}\right)\left(\begin{array}{c}
\hat{a}_{u} \\
\hat{a}_{l}
\end{array}\right)
$$

independently of the photon polarization [16.

Consider first the case of a $\sigma_{+}$-polarized photon entering the lower port of the first beam splitter, so that the initial state of the atom-field system is 


$$
\left|\psi_{\text {in }}\right\rangle=\hat{a}_{l,+}^{\dagger}|0\rangle\left|\phi_{\text {atom }}\right\rangle .
$$

As the photon propagates through the interferometer, Eqs. (2) and (1) show that the system evolves to the final state

$$
\begin{aligned}
\left|\psi_{\text {out }}\right\rangle & =-\frac{1}{2}|\mathcal{S}\rangle|g\rangle+\frac{i}{2 \sqrt{2}} \hat{a}_{l,+}^{\dagger}|0\rangle\left|m_{+}\right\rangle \\
& -\frac{1}{2 \sqrt{2}} \hat{a}_{u,+}^{\dagger}|0\rangle\left|m_{+}\right\rangle-\frac{1}{\sqrt{2}} \hat{a}_{u,+}^{\dagger}|0\rangle\left|m_{-}\right\rangle .
\end{aligned}
$$

Since the interferometer tuning is such that the upper detector $D_{u}$ clicks with unit probability in case no atom is present, the register of a click on the lower detector indicates with certainty the presence of an atom in the lower arm. As a result of the form of the interaction between the atom and the light field, this click can be interpreted as resulting from a "photon propagating through the upper arm", hence an IFM. Eq. (6) shows that the probability for such an event is $1 / 8$, a factor of 2 less than in the classical absorber and two-level atom cases. This is because in the present case, the atom can be thought of as a "half-absorber", a point to which we shall return shortly. After detection of a click on $D_{l}$, the normalized reduced atomic density operator is

$$
\rho_{\text {atom }, \text { out }} \propto \operatorname{Tr}_{\text {light }}\left\{\left|\psi_{\text {out }}\right\rangle\left\langle\psi_{\text {out }}\right| \hat{a}_{l,+}^{\dagger} \hat{a}_{l,+}\right\}=\left|m_{+}\right\rangle\left\langle m_{+}\right| .
$$

Hence, the IFM destroys the initial quantum superposition of the atomic state, leaving it in the energy eigenstate $\left|m_{+}\right\rangle$.

This results from the fact that an atom initially in state $\left|m_{-}\right\rangle$is transparent to $\sigma_{+}$-polarized light. Hence, if a signal is registered at detector $D_{l}$ we know for sure that the atom had to be initially in state $\left|m_{+}\right\rangle$. A measurement scheme using circularly polarized light therefore provides "which path" information about the atomic state, and leads to a projection of its state onto $\left|m_{+}\right\rangle$. Likewise, using $\sigma_{-}$-polarized light projects the superposition to $\left|m_{-}\right\rangle$ upon detection with $D_{l}$.

The "which path" information can be erased by using linearly polarized light, say along the $x$-direction. Decomposing this polarization into its circular components, the initial atom-field system is now in the state

$$
\left|\psi_{\text {in }}\right\rangle=\frac{1}{\sqrt{2}}\left(\hat{a}_{l,-}^{\dagger}-\hat{a}_{l,+}^{\dagger}\right)|0\rangle\left|\phi_{\text {atom }}\right\rangle,
$$

and yields the final state

$$
\begin{aligned}
\left|\psi_{\text {out }}\right\rangle & =-\frac{1}{2 \sqrt{2}}\left(|\mathcal{S}\rangle|g\rangle-\left|\mathcal{S}^{\prime}\right\rangle|g\rangle\right) \\
& +\frac{1}{4}\left(\hat{a}_{u,+}^{\dagger}|0\rangle\left|m_{+}\right\rangle-\hat{a}_{u,-}^{\dagger}|0\rangle\left|m_{-}\right\rangle\right) \\
& +\frac{1}{2}\left(\hat{a}_{u,+}^{\dagger}|0\rangle\left|m_{-}\right\rangle-\hat{a}_{u,-}^{\dagger}|0\rangle\left|m_{+}\right\rangle\right) \\
& -\frac{i}{4}\left(\hat{a}_{l,+}^{\dagger}|0\rangle\left|m_{+}\right\rangle-\hat{a}_{l,-}^{\dagger}|0\rangle\left|m_{-}\right\rangle\right) .
\end{aligned}
$$

It is the last term of this expression, the maximally entangled atom-photon state

$$
\left|\psi_{\text {out }}\right\rangle_{l} \propto \hat{a}_{l,+}^{\dagger}|0\rangle\left|m_{+}\right\rangle-\hat{a}_{l,-}^{\dagger}|0\rangle\left|m_{-}\right\rangle,
$$

which is of interest to us, since it is associated with the detection of light on the lower detector $D_{l}$. Karlsson and coworkers [8] discussed a similar state in the case of the IFM detection of a two-level atom and proposed its use to make a nondemolition measurement of the ground-state atom number. The present situation is different in that the entanglement is now in the state of the coupled atomfield system associated with the output at just one arm of the interferometer. As we now show, this entanglement can be used to perform an interaction-free, quantum nondemolition measurement of the quantum superposition of the atomic state. More generally, we can make use of the mapping of the atomic and photon states associated with the entanglement to encode an atomic superposition in the state of the measured photon. The associated selective measurement then leaves the atom in the desired quantum superposition.

We proceed by re-expressing the normalized state $\left|\Psi_{\text {out }}\right\rangle_{l}$ in terms of linearly polarized light components as

$$
\begin{aligned}
\left|\psi_{\text {out }}\right\rangle_{l} & =\frac{i}{\sqrt{2}} \hat{a}_{l, x}^{\dagger}|0\rangle\left(\left|m_{+}\right\rangle+\left|m_{-}\right\rangle\right) \\
& -\frac{1}{\sqrt{2}} \hat{a}_{l, y}^{\dagger}|0\rangle\left(\left|m_{+}\right\rangle-\left|m_{-}\right\rangle\right) .
\end{aligned}
$$

From this result it is immediately apparent that a polarization-sensitive measurement of $x$-polarized photons leaves the atoms in the pure final state

$$
\left|\phi_{\text {atom }}\right\rangle_{\text {out }}=\frac{1}{\sqrt{2}}\left(\left|m_{+}\right\rangle+\left|m_{-}\right\rangle\right),
$$

which is precisely the initial superposition $\left|\phi_{\text {atom }}\right\rangle$, while a detection of $y$-polarized photons gives the orthogonal final state

$$
\left|\phi_{\text {atom }}\right\rangle_{\text {out }}=\frac{1}{\sqrt{2}}\left(\left|m_{+}\right\rangle-\left|m_{-}\right\rangle\right) .
$$

In each case, the detection probability is readily seen from Eq. (9) to be 1/16, a factor of 2 less than previously and a factor of 4 less than in the case of two-level atoms. We are able to preserve the initial superposition because each circular polarized component performs an IFM on a different "half-absorber", located in the same interferometer path. The appropriate measurement of a linear polarization, a superposition of both circular polarizations, then combines both results.

It is important to remark that in the case of polarization-insensitive detection, the final atomic state is not a pure quantum superposition, but rather the mixture 


$$
\rho_{\text {atom }, \text { out }}=\frac{1}{2}\left(\left|m_{+}\right\rangle\left\langle m_{+}|+| m_{-}\right\rangle\left\langle m_{-}\right|\right) .
$$

We see, then, that the atom-photon entanglement between of the state $\left|\psi_{\text {out }}\right\rangle_{l}$ provides us with a tool not just to perform an interaction-free, quantum-nondemolition measurement of the state of an atom, but also to map its quantum coherence to a prescribed value.

We already mentioned that compared to the original EV scheme [1, the probability of detecting the presence of an atom in an interaction-free fashion, either without destroying its initial state or preparing it in a prescribed superposition, is considerably smaller than for two-level atoms. For the specific example considered here, the reduction is by a factor of 4 . This results from the multiplicity of atomic and light polarization states, i.e. the larger dimensionality of the relevant Hilbert space. Of all the "branches" followed by the wave function of the system during its evolution, only a few are useful to reconstruct the initial superposition state. The situation rapidly worsens for larger systems, and it is quite clear that decoherence-free IFMs soon become unrealistic. We note in addition that unlike in the EV scheme, there is no way to guarantee the preservation of the atomic state in case the photon is detected by the upper branch detector $D_{u}$. It is easily shown that in that case, the postmeasurement probability to find the atom in its initial state is always less than unity, due to the fact that there are two orthogonal maximally entangled contributions in Eq. (9) associated with the upper detector. Consequently, the initial state of the object has to be reset before a subsequent measurement can be performed, in contrast to the EV situation.

Despite these difficulties, it should be emphasized that the polarization-dependent measurement scheme presents advantages of considerable interest for quantum information processing applications. In particular, it is easily extended to the situation of entangled atoms, as illustrated in Fig. 2. Assume for concreteness that the two particles are initially in the Bell state

$$
\frac{1}{\sqrt{2}}\left(\left|m_{-}\right\rangle_{1}\left|m_{+}\right\rangle_{2}+\left|m_{+}\right\rangle_{1}\left|m_{-}\right\rangle_{2}\right)
$$

where $|\cdot\rangle_{i}$ corresponds to atom $i$. As a result of the local character of the measurement scheme, it follows that a choice of polarization-sensitive detection that preserves the quantum coherence of the atom inside the interferometer also preserves its entanglement with the other atom, while decoherence also implies the destruction of the entanglement. As such, decoherence-free IFMs provide a tool to monitor the presence of atoms without destroying their state of entanglement.

In summary, it is possible to determine the presence of an atom in a quantum superposition of internal states without destroying it, provided that the measurement scheme does not provide the "which way" information that would in principle permit to determine its internal state. This implies that when aimed at measuring multilevel atoms, IFMs have to be designed exceedingly carefully. Compared to the classical case their efficiency is very low, and information is lost even if the upper detector detects a photon. We also showed how polarizationsensitive IFMs can be used to map the polarization state of the detected photon onto the internal state of the atoms, and how these measurements translate directly to the domain of entangled atoms. Although our scheme does not actively prevent a system from decoherence, it opens up possibilities to better control quantum systems and monitor quantum systems, with potential applications in quantum information processing.

We wish to thank M. G. Moore and M. Wilkens for stimulating discussions. This work was supported in part by the Office of Naval Research Research Contract No. 14-91-J1205, the National Science Foundation Grant PHY98-01099, the US Army Research Office and the Joint Services Optics Program.

[1] A. C. Elitzur and L. Vaidman, Found. Phys. 23, 987 (1993).

[2] M. Renninger, Z. Phys. 158, 417 (1960).

[3] R. H. Dicke, Am. J. Phys. 49, 925 (1981).

[4] P. Kwiat, H. Weinfurter, T. Herzog, A. Zeilinger, and M. A. Kasevich, Phys. Rev. Lett 74, 4763 (1995).

[5] P. G. Kwiat, A. G. White, J. R. Mitchell, O. Nairz, G. Weihs, H. Weinfurter, and A. Zeilinger, Phys. Rev. Lett. 83, 4725 (1999).

[6] A. G. White, J. R. Mitchell, O. Nairz, and P. G. Kwiat, Phys. Rev. A 58, 605 (1998).

[7] M. Pavicic, Phys. Lett. A 223, 241 (1996).

[8] A. Karlsson, G. Björk, and E. Forsberg, Phys. Rev. Lett. 80, 1198 (1998).

[9] P. G. Kwiat, H. Weinfurter, and A. Zeilinger, in Coherence and Quantum Optics VII, 1996, edited by Eberly, Mandel, and Wolf, p. 673.

[10] A. G. White, P. G. Kwiat, and D. F. V. James, in Mysteries, Puzzles, and Paradoxes in Quantum Mechanics, 1999, edited by R. Bonifacio, p. 268.

[11] C. H. Bennett, G. Brassard, C. Crépeau, R. Jozsa, A. Peres, and W. K. Wootters, Phys. Rev. Lett 70, 1895 (1993).

[12] P. Shor, in Proceedings 35th Annual Symposium on Foundations of Computer Science, 1994, p. 124.

[13] J. Cirac and P. Zoller, Phys. Rev. Lett 74, 4091 (1995).

[14] A. Ekert, Phys. Rev. Lett. 67, 661 (1991).

[15] J. Rarity, P. Ownes, and P. Tapster, J. Mod. Opt. 41, 2435 (1994).

[16] L. Mandel and E. Wolf, Optical Coherence and Quantum Optics (Cambridge University Press, 1995). 